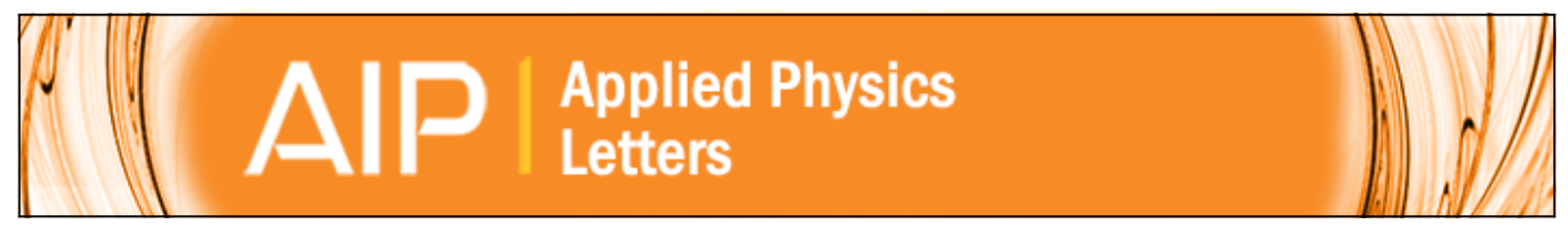

\title{
Acoustic-counterflow microfluidics by surface acoustic waves
}

Marco Cecchini, Salvatore Girardo, Dario Pisignano, Roberto Cingolani, and Fabio Beltram

Citation: Applied Physics Letters 92, 104103 (2008); doi: 10.1063/1.2889951

View online: http://dx.doi.org/10.1063/1.2889951

View Table of Contents: http://scitation.aip.org/content/aip/journal/apl/92/10?ver=pdfcov

Published by the AIP Publishing

\section{Articles you may be interested in}

Adjustable, rapidly switching microfluidic gradient generation using focused travelling surface acoustic waves Appl. Phys. Lett. 104, 023506 (2014); 10.1063/1.4862322

Density-dependent separation of encapsulated cells in a microfluidic channel by using a standing surface acoustic wave

Biomicrofluidics 6, 024120 (2012); 10.1063/1.4718719

Shear horizontal surface acoustic wave induced microfluidic flow

Appl. Phys. Lett. 99, 153704 (2011); 10.1063/1.3651487

Streaming phenomena in microdroplets induced by Rayleigh surface acoustic wave J. Appl. Phys. 109, 114901 (2011); 10.1063/1.3586040

Double aperture focusing transducer for controlling microparticle motions in trapezoidal microchannels with surface acoustic waves

Appl. Phys. Lett. 95, 134101 (2009); 10.1063/1.3238313

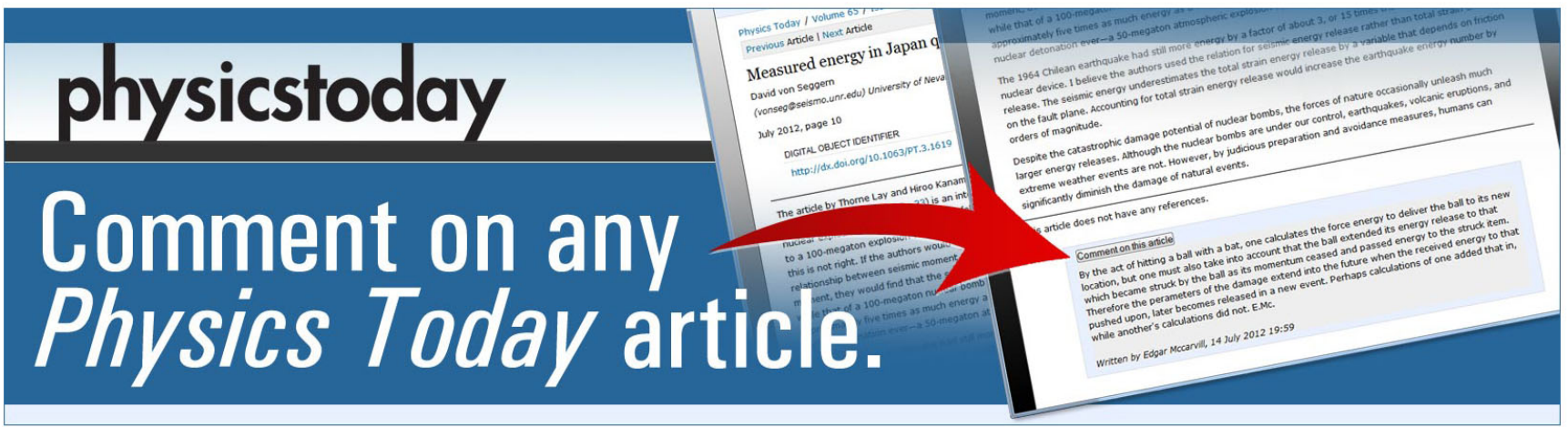




\title{
Acoustic-counterflow microfluidics by surface acoustic waves
}

\author{
Marco Cecchini ${ }^{1,2, a)}$ Salvatore Girardo, ${ }^{3,4}$ Dario Pisignano, ${ }^{3,4}$ Roberto Cingolani, ${ }^{3,4}$ and \\ Fabio Beltram ${ }^{1,2}$ \\ ${ }^{1}$ Scuola Normale Superiore and Italian Institute of Technology, I-56126 Pisa, Italy \\ ${ }^{2}$ Scuola Normale Superiore and NEST-CNR-INFM, I-56126 Pisa, Italy \\ ${ }^{3}$ National Nanotechnology Laboratory (NNL) of CNR-INFM, Università degli Studi del Salento, \\ clo Distretto Tecnologico ISUFI, via Arnesano, I-73100 Lecce, Italy \\ ${ }^{4}$ Fondazione Istituto Italiano di Tecnologia (IIT), via Morego 30, I-16163 Genova, Italy
}

(Received 10 October 2007; accepted 7 February 2008; published online 10 March 2008)

\begin{abstract}
In this letter, we demonstrate an unexpected surface-acoustic-wave (SAW)-driven pumping effect in hydrophobic polydimethilsiloxane (PDMS)-lithium niobate $\left(\mathrm{LiNbO}_{3}\right)$ microchannels. Atomization within the fluidic channel followed by SAW-assisted coalescence leads to liquid counterflow with respect to the SAW propagation direction. This physical mechanism is contrasted with the acoustic-streaming process driving isolated drop displacement on piezoelectric substrates. This principle is shown not to be readily applicable to the present microchannel case. The proposed device geometry can be exploited to integrate micropumps into complex microfluidic chips, improving the portability of micro-total-analysis systems. (C) 2008 American Institute of Physics.

[DOI: 10.1063/1.2889951]
\end{abstract}

The increasing demand for low-cost and portable devices for biomedical applications has stimulated the development of advanced micro-total-analysis systems. ${ }^{1}$ Typical analysis and reaction processes benefit from device miniaturization since this yields efficient and reliable readout with very small sample and reagent quantities. Indeed, during the last few years, microfluidics has become a very active research area ${ }^{2,3}$ and has stimulated progress in materials science and nonconventional micro-/nanofabrication technologies. ${ }^{4}$

A very original approach to microfluidics was recently introduced by Wixforth et al. ${ }^{5,6}$ This is based on a microfluidic system fully controlled by surface acoustic waves (SAWs) that avoids all pumping difficulties related to small pipe networks. ${ }^{7}$ These authors were able to replace closed fluidic channels by hydrophilic traces on a chip surface: the liquid was transported in single droplets by the SAW-fluid interaction. Specifically, when a traveling SAW interacts with a liquid droplet placed on a free surface, its compressional component is diffracted at the Rayleigh angle into the droplet, generating a leaky SAW (LSAW) (Ref. 8) phenomenon caused by SAW-radiation leakage into the liquid. The LSAW component generates a pressure gradient within the droplet that results in the so-called acoustic streaming, i.e., momentum and energy transfer from the wave to the liquid. This gives rise to a liquid response that depends on the SAW power. ${ }^{8}$ For high-power excitation, jet propulsion or atomization can occur. ${ }^{9}$ At low excitation power, efficient mixing is easily obtained, whereas at intermediate excitation powers, the entire droplet translates along the direction of the SAW propagation. SAW-driven transport of droplets confined between two planes was also demonstrated. ${ }^{8}$ In the case of a $300 \mu \mathrm{m}$ gap between the planes and a SAW wavelength of $180 \mu \mathrm{m}$, Renaudin et al. showed that net displacement along the SAW propagation direction can be obtained. ${ }^{8}$ They also showed that in case of absence of surface functionalization, acoustic streaming leads to wavy, inaccurate droplet trajec-

\footnotetext{
${ }^{\text {a)} E l e c t r o n i c ~ m a i l: ~ m . c e c c h i n i @ s n s . i t . ~}$
}

tories. At present, virtually all microfluidic systems being used or developed are based on microchannels. ${ }^{3}$ In all these systems, liquid pumping is provided by displacement or dynamic micropumps ${ }^{10}$ often based on off-chip elements.

In this letter, we investigate the applicability of SAWbased pumping to microchannel-based fluidic systems and demonstrate an unexpected SAW-driven operation based on a physical mechanism completely different from acoustic streaming. We show that atomization within the fluidic channel followed by SAW-assisted coalescence can lead to liquid counterflow with respect to the SAW propagation direction. This mechanism yields efficient water injection into polydimethilsiloxane (PDMS)- $\mathrm{LiNbO}_{3}$ microchannels at a controllable rate in line with the other available pumping methods. ${ }^{10}$

We employed a combination of photo- and soft lithography ${ }^{11}$ to fabricate devices with different fluidic geometries. The basic layout consists of two layers. The bottom layer is a $\mathrm{LiNbO}_{3}$ piezoelectric substrate, with two microfabricated interdigital transducers (IDTs) for SAW excitation and detection. The IDTs were composed by 20 pairs of 500- $\mu \mathrm{m}$-long Al fingers with $24 \mu \mathrm{m}$ periodicity $(\sim 160 \mathrm{MHz}$ resonance frequency on $\mathrm{LiNbO}_{3}$ ), placed at a distance of $3.4 \mathrm{~mm}$. The upper layer is a patterned PDMS film. Microchannels were obtained in the film by replica molding. ${ }^{12}$ Channel geometries with lateral dimensions between 120 and $520 \mu \mathrm{m}$ and relative heights between 10 and $50 \mu \mathrm{m}$ were transferred onto PDMS replicas. Final devices were straightforwardly assembled by conformal bonding ${ }^{12}$ of the two layers [Fig. 1(a)] and provided alignment of the polymeric stamps with the IDTs [Fig. 1(b)]: the hybrid microchannels were defined by the $\mathrm{LiNbO}_{3}$ bottom wall and the PDMS lateral and top walls.

Fluid dynamics was monitored by a Leica MZ16 stereomicroscope coupled to a Basler A602f-2 fast acquisition camera (400 frames/s at $640 \times 120$ pixel resolution) with a direct hard-disk writing system for long-time recording by Advanced Technologies. Video postprocessing was carried out by VIRTUALDUB software. ${ }^{13}$ The sample temperature was 

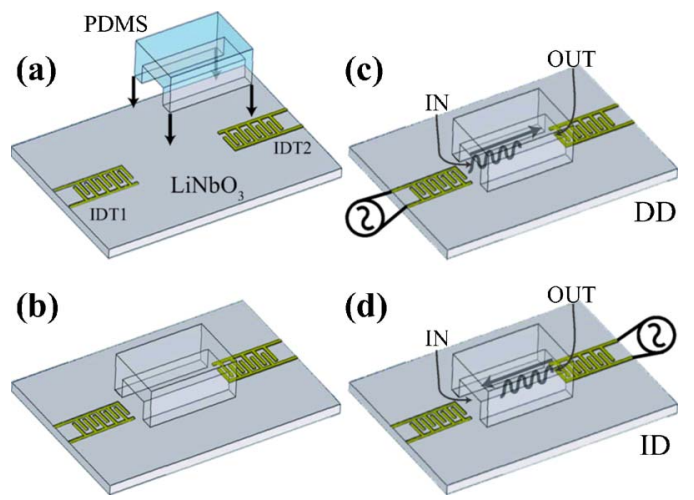

FIG. 1. (Color online) Scheme for the assembling of microfluidic devices and the activation of the liquid motion into microchannels: [(a) and (b)] final devices were fabricated by the superposition of a PDMS textured element and a $\mathrm{LiNbO}_{3}$ substrate layer with IDTs for SAW excitation and detection; (c) DD and (d) ID experimental arrangements.

set by a Thorlabs TED350 temperature controller connected to a Peltier refrigerator. The measurements reported here refer to a representative device, with IDTs operating at $160 \mathrm{MHz}$ resonance frequency and coupled to a 1.1-mm-long fluidic channel with a $210 \times 20 \mu \mathrm{m}^{2}$ section. All the devices studied, apart from the influence of the geometrical details, exhibited the same fluidic behavior.

For a preliminary experiment, we released a de-ionized water drop of about $2 \mu \mathrm{l}$ at the entrance of the microchannel by a micropipet and monitored any filling by capillarity. As expected, the liquid did not enter the microchannel owing to the hydrophobicity of untreated PDMS surfaces. ${ }^{14,15}$ Continuous SAWs were then excited, and the position of the water-air interface within the channel was monitored as a function of time and power of the signal applied to the IDT $\left(P_{\mathrm{SAW}}\right) . P_{\mathrm{SAW}}$ was limited to $20 \mathrm{dBm}$ in order to prevent substrate damage. We analyzed two different experimental arrangements. First, SAWs were excited from one IDT to the channel entrance and, hence, along the channel toward its outlet [direct drive (DD)] [Fig. 1(c)]. Second, the SAWs were launched in the opposite direction, i.e., from the other IDT, so that SAWs propagated from the channel outlet toward its inlet [inverted drive (ID)] [Fig. 1(d)].

In case of conventional DD, with increasing $P_{\mathrm{SAW}}$, we observed the expected droplet deformation caused by acoustic streaming ${ }^{16}$ and, finally, a rather slow movement of the liquid into the channel $\left(P_{\mathrm{SAW}} \approx 20 \mathrm{dBm}\right)$ [Fig. 2(a)]. At these power values, however, significant droplet atomization occurred that strongly affected the droplet outside the channel, where incoming SAW power was maximum. This led to fast evaporation of the water reservoir and prevented the filling of the microchannel [Fig. 2(a)]. For the present experiments, the water reservoir ran out in $\sim 9 \mathrm{~s}$, avoiding the injection of amount of liquid larger than $0.8 \mathrm{nl}$ (i.e., about $200 \mu \mathrm{m}$ of meniscus travel). As expected, SAW-liquid drag was observed when liquid evaporation led to the formation of isolated droplets within the microchannel [Fig. 2(a); $t=9.63 \mathrm{~s}$, $t=10.50 \mathrm{~s}]$ or when the liquid drop was released away from the microchannel (not shown).

ID showed a very different behavior. For $P_{\mathrm{SAW}}$ $>14 \mathrm{dBm}$, a fast liquid transfer from the reservoir droplet into the microchannel [Fig. 2(b)] was observed. We stress that the liquid was driven in the opposite direction with respect to the SAW propagation direction. In view of the

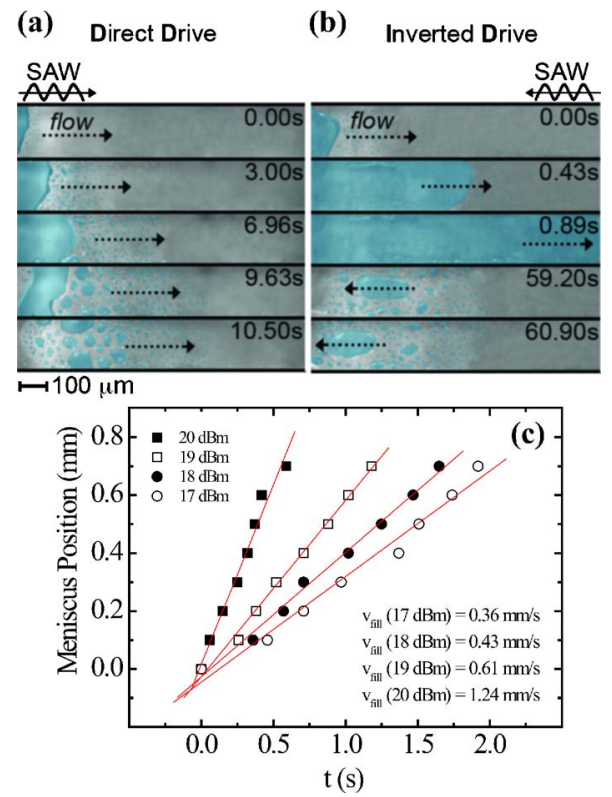

FIG. 2. (Color online) (a) Photographs of the water filling process for DD configuration at different times $(t)$. The SAW was turned on (IDT1) at $t=0.00$. After $6.96 \mathrm{~s}$, the water reservoir at the channel entrance was completely evaporated. (b) Filling process for ID: the SAW was excited (IDT2) at $t=0.00 \mathrm{~s}$. After $0.89 \mathrm{~s}$ the microchannel was completely filled by water. In the last two frames, the water reservoir was evaporated and the drops present in the microchannel moved in the same direction as SAW propagation. (c) Meniscus position as a function $t$ for ID configuration at different $P_{\text {SAW. }}$

known SAW-fluid interaction properties, ${ }^{5,8}$ so far leading to liquid drag only along the SAW direction, this phenomenon is quite unexpected and, to the best of our knowledge, has never been observed before. Figure 2(c) displays the meniscus position versus time at different $P_{\text {SAW }}$ values and demonstrates a controllable and fast filling dynamics. Filling velocity ${ }^{17}\left(v_{\text {fill }}\right)$ could be controlled up to a maximum value of $1.24 \mathrm{~mm} / \mathrm{s}$ (i.e., $\sim 0.3 \mu \mathrm{l} / \mathrm{min}$ for the present channel geometry) by varying $P_{\text {SAW }}$ up to $20 \mathrm{dBm}$, yielding rapid filling $(t=0.9 \mathrm{~s})$ of the whole channel, without significant evaporation of the droplet reservoir [Fig. 2(b)]. These values are in line with what were reported in the literature for pumping mechanisms compatible with lab-on-chip technologies. ${ }^{10}$

ID pumping is indeed surprising in light of the fact that any momentum transfer to the liquid must be in the opposite direction with respect to actual fluid flow. Conventional acoustic-streaming physics, therefore, does not apply. In order to understand this dramatic difference between the ID and DD behaviors we must consider the different positions where the SAW-liquid interaction occurs. In the ID configuration, the interaction is maximum within the capillary and leads to a drastically enhanced water nebulization rate at the meniscus position. The impact of this atomization is analyzed in Fig. 3, in which a selected sequence of formation and growth of water particles sprayed off the main fluid drop within the channel is shown. Evolution of these droplets and their interaction with the liquid meniscus determine the observed pumping phenomenon. Figure 3 shows continuous small droplet generation and coalescence followed by merging with the meniscus. The latter phenomenon changes the position of the liquid-air interface, resulting in a net fluid movement in the opposite direction with respect to SAW 


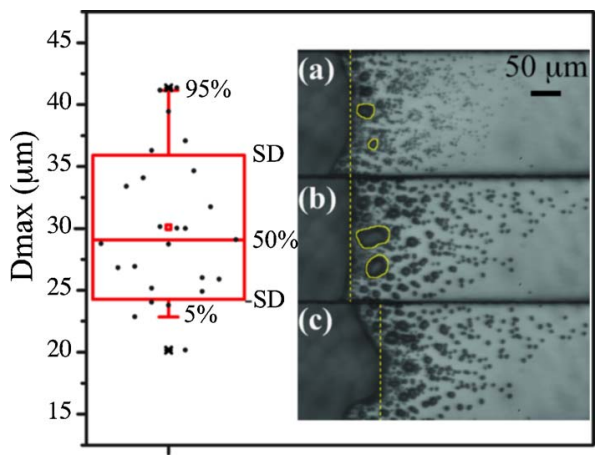

FIG. 3. (Color online) Right panel: nebulization detail for ID configuration. The droplets grow in volume and finally merge with the liquid meniscus, leading to a net displacement of the liquid. Left panel: statistical analysis of measured droplets diameter at coalescence. Droplet diameter is reported as $10 \%-90 \%$ distribution (a random sample of the population is plotted as scattered black points). The population mean is reported as a horizontal red line enclosed in a rectangular box whose vertical length represents the standard deviation of the mean.

propagation. Meniscus advancement requires this coalescence. Interestingly, merging of the droplet with the water bulk is favored by the SAW itself since acoustic-streamingdriven momentum transfer to the individual droplets pushes them toward the meniscus. It is known that only droplets with lateral dimension greater than the SAW wavelength can be affected by acoustic-streaming energy transfer. ${ }^{5}$ Data presented in Fig. 3 demonstrate that the droplet diameter before coalescence $(30 \pm 6 \mu \mathrm{m})$ is comparable to the SAW wavelength $(24 \mu \mathrm{m})$. This confirms that acoustic counterflow is promoted by standard acoustic-streaming drag.

It is interesting to compare the present phenomenon to recent results reported by Liu et al. ${ }^{18}$ These authors generated small droplets at the liquid-air interface within a hydrophobic microchannel by means of laser-induced local heating. Also, in this case, droplet coalescence with the bulk of the liquid led to the translation of the water meniscus.

Conventional SAW-driven transport (i.e., the liquid moves along the SAW direction) was observed within the channel in case of isolated droplets also for the ID configuration. For example, following the phase discussed so far with channel filling, continued SAW application leads to water evaporation and exhaustion of the water reservoir at the channel inlet. Isolated water spots form in the capillary and standard SAW drag occurred, leading to the inversion of the water motion [fourth and fifth frames of Fig. 2(b)]. This behavior agrees with the SAW-driven transport of droplets confined between two planes already observed by Renaudin et al. ${ }^{8}$

In conclusion, we demonstrated efficient SAW-driven liquid pumping into hybrid $\mathrm{PDMS} / \mathrm{LiNbO}_{3}$ microchannels. We showed that conventional acoustic-streaming pumping cannot be straightforwardly applied in the case of microchannel-based systems and presented an alternative counterpropagation regime named ID. ID led to controlled liquid transport along the opposite direction with respect to the SAW propagation with velocities up to $1.24 \mathrm{~mm} / \mathrm{s}$ (i.e., $\sim 0.3 \mu \mathrm{l} / \mathrm{min}$ for the present channel geometry) by varying $P_{\text {SAW }}$ up to $20 \mathrm{dBm}$.

ID appears to be very promising for the fabrication of integrated micropumps for microfluidic chips and micrototal-analysis systems. Indeed, the present approach requires only an external signal generator set at the IDT resonance frequency. Importantly, impedance matching and device geometry optimization (i.e., channel shape, IDT periodicity/ aperture, IDT position, etc.) should allow battery operated systems. Work is in progress to extend this approach to more complex microfluidic networks by integrating several IDTs on the same chip to drive fluids along specific paths.

This work was supported in part by MIUR under the FIRB Project Nos. RBLA03ER38 and RBIN045NMB.

${ }^{1}$ K. A. Shaikh, K. S. Ryu, E. D. Goluch, J.-M. Nam, J. Liu, C. Shad Thaxton, T. N. Chiesl, A. E. Barron, Y. Lu, C. A. Mirkin, and C. Liu, Proc. Natl. Acad. Sci. U.S.A. 102, 9745 (2005); P. S. Dittrich, K. Tachikawa, and A. Manz, Anal. Chem. 78, 3887 (2006).

${ }^{2}$ J. C. T. Eijkel and A. van den Berg, Microfluid. Nanofluid. 1, 249 (2005);

J. Atencia and D. J. Beebe, Nature (London) 437, 648 (2005).

${ }^{3}$ T. M. Squires and S. R. Quake, Rev. Mod. Phys. 77, 977 (2005).

${ }^{4}$ J. C. T. Eijkel and A. van den Berg, Lab Chip 5, 1202 (2005); M.-S. Kim,

S. I. Cho, K.-N. Lee, and Y.-K. Kim, Sens. Actuators B 107, 818 (2005).

${ }^{5}$ A. Wixforth, C. Strobl, Ch. Gauer, A. Toegl, J. Scriba, and Z. v. Guttenberg, Anal. Bioanal. Chem. 379, 982 (2004).

${ }^{6}$ K. Sritharan, C. J. Strobl, M. F. Schneider, A. Wixforth, and Z. Guttenberg, Appl. Phys. Lett. 88, 054102 (2006).

${ }^{7}$ G. M. Whitesides, Nature (London) 442, 368 (2006).

${ }^{8}$ A. Renaudin, P. Tabourier, V. Zhang, J. C. Camart, and C. Druon, Sens. Actuators B 113, 389 (2006).

${ }^{9}$ M. Kurosawa, T. Watanabe, A. Futami, and T. Higuchi, Sens. Actuators, A 50, 69 (1995); K. Chono, N. Shimizu, Y. Matsui, J. Kondoh, and S. Shiokawa, Jpn. J. Appl. Phys., Part 1 43, 2987 (2004).

${ }^{10}$ D. J. Laser and J. G. Santiago, J. Micromech. Microeng. 14, R35 (2004).

${ }^{11}$ Y. N. Xia and G. M. Whitesides, Angew. Chem., Int. Ed. 37, 551 (1998).

${ }^{12}$ J. M. Ng, I. Gitlin, A. D. Stroock, and G. M. Whitesides, Electrophoresis 23, 3461 (2002)

${ }^{13}$ D. Pisignano, F. Di Benedetto, L. Persano, Synth. Met. 153, 325 (2005); D. Pisignano, E. Sariconi, M. Mazzeo, G. Gigli, and R. Cingolani, Adv. Mater. (Weinheim, Ger.) 14, 1565 (2002).

${ }^{14}$ Modification of wettability is possible [H. Makamba, J. H. Kim, K. Lim, N. Park, and J. H. Hahn, Electrophoresis 24, 3607 (2003)] and microchannel filling can be obtained by spontaneous capillarity, but PDMS hydrophilic state is not stable and hydrophobicity is recovered by exposition to air [I. J. Chen and E. Lindner, Langmuir 23, 3118 (2007)].

${ }^{15}$ S. J. Clarson and J. A. Semlyn, Siloxane Polymers (Prentice Hall, New York, 1993).

${ }^{16}$ D. Beyssen, L. Le Brizoual, O. Elmazria, and P. Alnot, Sens. Actuators B 118, 380 (2006)

${ }^{17} v_{\text {fill }}$ is defined as the slope of the linear curve fit of meniscus position as a function of time data.

${ }^{18}$ G. L. Liu, J. Kim, Y. Lu, and L. P. Lee, Nat. Mater. 5, 27 (2006). 